\title{
Whole Grains in Amelioration of Metabolic Derangements
}

\author{
Samir Develaraja ${ }^{1}$, Anup Reddy², Mukesh Yadav ${ }^{3}$, Shalini Jain ${ }^{4}$, Hariom Yadav** \\ ${ }^{1}$ University of Pennsylvania, College of Arts and Sciences, Philadelphia, PA. \\ ${ }^{2}$ West Virginia University School of Medicine, Robert C. Byrd Health Sciences Center, Morgantown, USA. \\ ${ }^{3}$ College of Advance Studies, Datia, M.P, India. \\ ${ }^{4}$ National Institute of Diabetes and Digestive and Kidney Diseases, National Institutes of Health, Bethesda, USA
}

Received: August 29, 2016; Accepted: October 14, 2016; Published: October 24, 2016

*Corresponding author: Dr. Hariom Yadav, Diabetes, Endocrinology and Obesity Branch, National Diabetes and Digestive and Kidney Diseases, National Institutes of Health, Bethesda, MD 20892, USA. E-mail: yadavhariom@gmail.com

\begin{abstract}
Daily diet influences whole body metabolism, and intricately linked to the prevention or progression of metabolic diseases including obesity, diabetes and cardiovascular diseases. Several epidemiological and large scale studies have shown that diets enriched with whole grains improves metabolic function and protect from the development of metabolic diseases. Direct impact of whole grain diet can be mediated on several levels of metabolic functions i.e. reduced glycemic index, improved fat oxidation potential, increased cholesterol clearance or decreased cholesterol biosynthesis and modulation of gut microbiome. In this article we reviewed several studies indicating the beneficial effects of whole grain diets on metabolic functions, as well as discussed the potential active phytochemicals present in these whole grain foods to contribute in modulation of metabolic function in our body.
\end{abstract}

Keywords: Whole Grain; Diabetes; Obesity; Metabolism; Fat; Phytochemicals; Nutrition; Microbiome; Metabolic Diseases;

\section{Introduction}

The sharp spike in the prevalence and severity of obesity, type 2 diabetes and metabolic syndrome is a chief concern for society as a whole [1]. Obesity is characterized by the accumulation of excess fat in the adipose tissue. At its core, the obesity epidemic stems from an excess of energy intake compared to energy expenditure [1]. Thus, consumption of "healthy" diet is a critical to curtailing the dual epidemics of obesity and type 2 diabetes. Currently, a tremendous amount of research worldwide explores the beneficial health effects and nutritional composition of foods that are part of our diet. Not only vitamins and minerals are explored for their promised beneficial properties, but also bioactive phytochemicals in functional foods are of great interest in research. Growing evidence asserts potent roles of phytoconstituents of various functional foods in the amelioration of chronic diseases, including diabetes, obesity and the resultant metabolic syndrome $[2,3]$.
Whole grains were brought to public attention as research around the world to uncover their beneficial health effects. The 1995 USDA Dietary Guidelines did not specifically recommend the intake of whole grains; however, the 2005 and 2010 guidelines suggested consuming at least 3 ounces of whole grains per day, and ensuring $50 \%$ of daily grain intakes come from whole grains [4,5]. Further, a markedly greater number of whole grain products are available to consume in the current market. The most recent examination of the principal sources of whole grains in the American diet was done by Bachman et al. in 20012002 [6]. Wheat is the leading grain in the American diet, and oats, corn, barley, rice, rye, millet and spelt are all consumed in varying proportions $[6,7]$. These whole grains are present in many types of foods like breads, pastas, baked goods, tortillas, breakfast cereal and several others.

The broad spectrum of foods that constitute whole grains, coupled with varying definitions of a "whole grain" between organizations, studies and databases renders much uncertainty in the estimation of an individual's whole grain intake. Regardless of the variance, however, the overwhelming majority of studies assert that majority of population with western food habits do not consume adequate amounts of whole grains. Despite the positive steps taken by governmental agencies and industry, the average Americans still does not consume the recommended amount of whole grains per day [8-10].

\section{Whole Grains}

According to the FDA and American Association of Cereal Chemists International, a whole grain is defined as "whole grain consists of the intact, ground, cracked or flaked caryopsis, whose principal anatomical components - the starchy endosperm, germ and bran - are present in the same relative proportions as they exist in the intact caryopsis" [11]. A whole grain may either remain intact, or be reconstituted. A reconstituted whole grain is one in which the components of the whole grain are recombined 
to match relative proportions that are present in the naturally occurring kernel [12]. The overwhelming majority of whole grain products commercially distributed fall under the category of reconstituted whole grains.

The outermost layer of a whole grain is the bran, which contains mainly non-digestible carbohydrates (cellulose, arabinoxylan and others). Function of bran is to protect the inner layers from external environmental stresses. The inner layers of the grain - the endosperm and the germ consist of soluble fibers, resistant starch, vitamins, minerals, and various other phytonutrients (polyphenols and others) [11]. Refining whole grains removes the bran and germ, leaving the endosperm to be processed into white flour (Figure 1). The majority of nutrients and phytochemicals, however, are concentrated in the bran and germ portions of the whole grain $[13,14]$. Therefore, many of the key nutrients and phytochemicals, including over $75 \%$ of the fibers, are physically removed through the process of refining [15]. Overall, whole grains have higher phytonutrient content, leading to higher antioxidant activity, and more beneficial modulation of glucose and energy homeostasis. Refining wheat loses $83 \%$ of total phenolic acids, $79 \%$ of total flavonoids, $93 \%$ of ferulic acid, $78 \%$ of total zeaxanthin, $51 \%$ of total lutein, and $42 \%$ of total $\beta$-cryptoxanthin [16]. Table 1 described major macronutrient composition of the most common whole grains (Table 1)

\section{Epidemiology: Benefits of Whole Grains In Diabetes And Metabolic Diseases}

The epidemiology demonstrating that consumption of whole grains ameliorates obesity, type II diabetes and their resultant complications is fairly consistent. Over the past decade, many large scale studies have reached the conclusion that there exists a significant inverse association between the intake of whole grains and the incidence of type II diabetes [14] and cardiovascular disease in both sexes [17-19]. Kastorini and Panagiotakos reviewed several broad studies exploring whole grains and diabetes [20] and Priebe et al. compiled numerous cohort studies $[21,22]$. Phytochemicals present in whole grains may confer

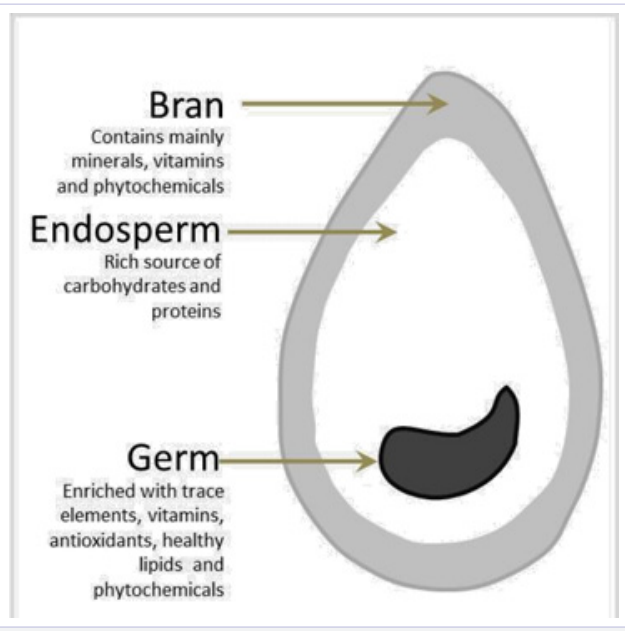

Figure 1: Anatomy of a whole grain and major ingredients. potent anti-diabetic/obesity properties. The succeeding sections of this article will review the important phytochemicals part of whole grains, and their role in amelioration of type 2 diabetes and metabolic derangements. However, as the research exploring the specific effects of phytochemicals isolated from whole grains is relatively young, it is important to keep the findings in context until stronger and more mechanistic studies demonstrate their definite role against metabolic diseases. Furthermore, as it has been shown with phytochemicals from fruits, vegetables and other food groups have synergistic effect of whole grain's phytochemicals that may be responsible for the significant health benefits derived from whole grains.

\section{Whole Grains And Blood Glucose Regulation}

Pioneering studies conducted in 1980's uncovered the role of whole grains in the regulation of glucose homeostasis. Compared to a high glycemic index diet ( $\mathrm{GI}=90)$ of potatoes and white bread, a low glycemic index $\operatorname{diet}(\mathrm{GI}=67)$ containing bread with whole grains, parboiled wheat, pasta and legumes demonstrated a significantly decreased blood glucose and circulating insulin levels in both normal and diabetic subjects [23]. And compared with a diet using refined grain flour, one using whole grain flour demonstrated a significantly lowered circulating glucose and insulin levels [24]. In 2004, Slavin thoroughly reviews the subsequent studies that substantiated the evidence of association between whole grain intake and beneficial regulation of glucose homeostasis, and in some cases, amelioration of diabetes mellitus [25]. Overall, they reports that consumption of whole grains is inversely correlated with circulating insulin and glucose levels. Interestingly in 2002, Periera et al. showed that there may be enhancement of insulin sensitivity due to whole grain intake [26].

The research about whole grains and metabolic diseases is young, and relatively few phytochemicals from whole grains have been shown to beneficially from modulate glucose metabolism. Many anti-nutrients, including phytic acid, amylase inhibitors, saponins, phenolics and lectins have been shown to decrease circulating glucose and insulin [25]. Cyanidin, an anthocyanidin with a characteristic reddish-orange color, has previously been shown to reduce blood glucose levels and improve insulin sensitivity due to the reduction of retinol binding protein 4 expression in type 2 diabetic mice [27]. In addition,

Takanori et al discovered that cyanidin glucoside from purple corn extract has a potential to ameliorate diabetic complications [28]. Members of the carotenoids, flavonoids, hydroxycinnamic acids, vitamin E, and other phytochemicals that are part of whole grains have also been shown to play beneficial roles in diabetes. $\beta$-Cryptoxanthin is an antioxidant, which may help prevent free radical damage to cells and DNA, as well as to stimulate the repair of oxidative damage to DNA. An increased intake of total $\beta$-Cryptoxanthin along with other phytochemicals was associated with a reduced risk to type 2 diabetes $[29,30]$. Lutein, an antioxidant that is an essential chemical in ocular function, is also found in whole grains [31]. Arnal et al found that a combined treatment with lutein and insulin prevented the development of cataracts in streptozotocin-induced diabetic 


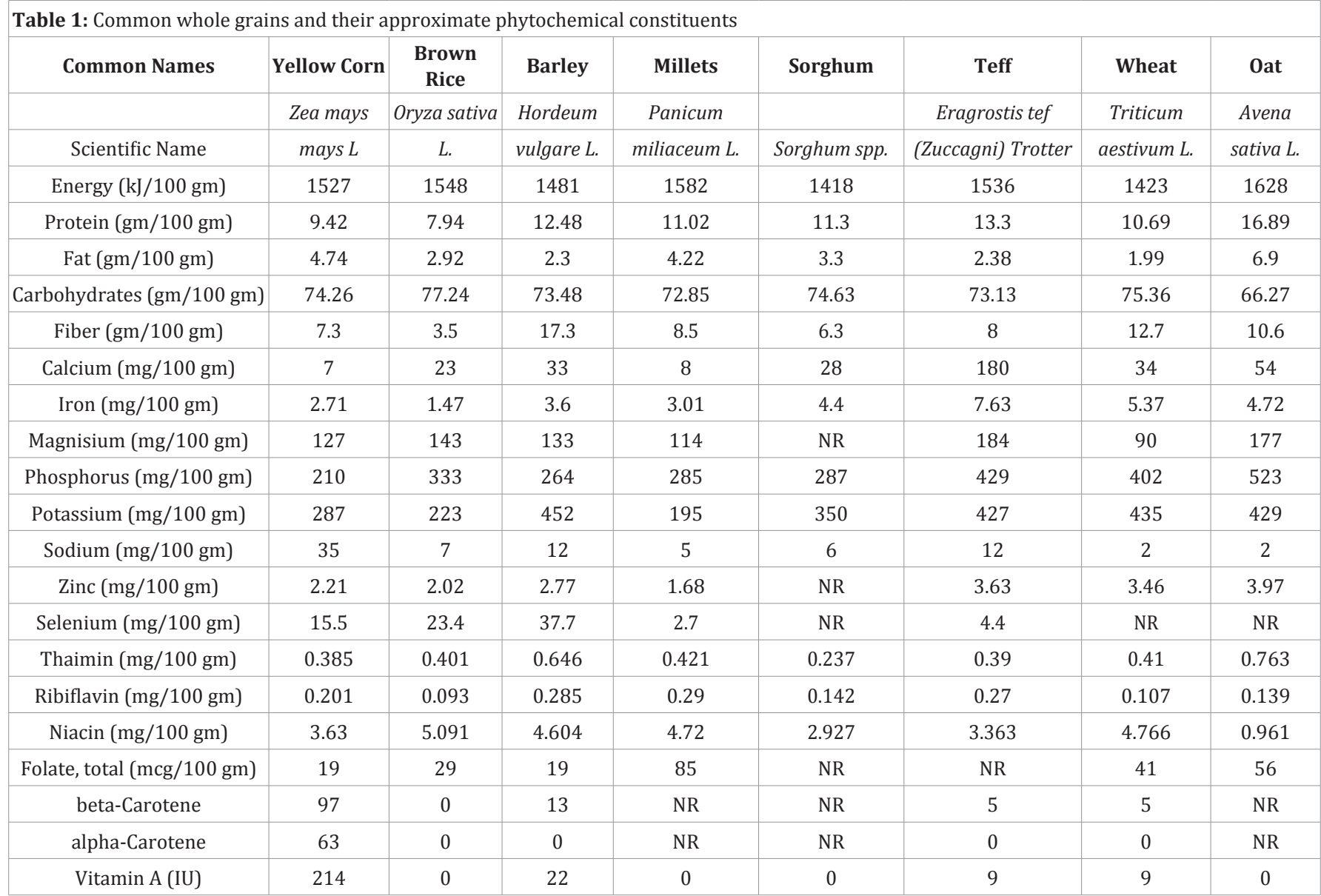

rats by inhibiting lipid peroxidation in the eye lenses of diabetic rats [31]. Furthermore, Muriach et al discovered that lutein may reduce susceptibility to infections in diabetic patients [32], as diabetic subjects have higher risk of common infections [33, 34]. Another phytonutrient present in whole grains, zeaxanthin, also has the potential to inhibit the development of retinopathy in diabetic patients [35].

Acute consumption of barley $\beta$-glucan, but not resistant starch, in muffins was effective in reducing glucose and insulin responses in men who were mildly insulin-resistant [36-38].

Dietary intake of $\beta$-glucans has been shown to help with control of blood glucose level and lipids; and reduction of hypertension [39]. Kobori et al asserted that quercetin (a flavonol found in many fruits, vegetables, leaves and grains) reduced blood glucose level and improved plasma insulin levels in streptozotocin-induced diabetic rats [40]. Another study concluded that quercetin may have a pharmacological application in treating cardiovascular disease in diabetic patients via its antioxidant and anti-inflammatory potential [41, 42]. Yang et al showed that quercetin combined with resveratrol can inhibit fat cell differentiation and development [43].

Ohnishi et al found that dietary ferulic acid can alleviate oxidative stress and attenuated hyperglycemic associated response in diabetes [44]. Balasubashini et al showed that ferulic acid enhances antioxidant capacity thus alleviating diabetes [45].

In another study, it has been shown that saponin (a common phytochemical found in grains) from Tribulue terrestris could significantly reduce the level of serum glucose [46], but it was not clear that saponin from whole grain sources has the same effects on blood glucose levels. Lower $\beta$-tocopherol (vitamin E) concentration is known to be associated with impaired insulin sensitivity $[47,48]$, and treatment of vitamin E exerts a protective role against diabetes-induced peripheral muscle dysfunction and renal function [49-51], as well as reduced risk of type 2 diabetes [30]. Kushad et al showed that tocotrienol can prevent diabetes associated cognitive deficits [52], and reduced risk of type 2 diabetes have been associated with increased tocotrienol [30]. Mice on diets with greater phytic acid (a common anti-nutrient in grains) intake displayed lower blood glucose levels after glucose tolerance tests $[53,54]$, and also known to lower blood glucose response by reducing the rate of starch digestion and slowing the gastric emptying [55]. Gamma-oryzanol or rice bran oil (an oil extracted from the hard outer brown layer of rice after chaff / rice husk) increased insulin sensitivity in diabetic mice [56]. Despite the current research efforts, there is a dearth of studies that explore the molecular mechanisms and the role of specific whole grain phytochemicals to regulate glucose homeostasis. Although 
many hypotheses (e.g. the fiber hypothesis) float around, but it is critical that future research explores the beneficial effects of whole grains in blood sugar metabolism at a mechanistic level. Further, as many believe that the mechanism by which whole grains regulate glucose metabolism is largely based on the botanical structure of the grain and its phytochemicals; detailed analyses of structural interactions are warranted. Overall, it is critical to further elucidate the role of whole grain phytochemicals in ameliorating the pathogenesis of type 2 diabetes.

\section{Antioxidant Activity of Whole Grains}

Antioxidant activity is a potent mechanism by which whole grains deliver their beneficial health effects against several human diseases including obesity and diabetes. At core, the function of antioxidants is to react with free radicals to protect against free radical or Reactive Oxygen Species (ROS) attack on self-lipids, protein and DNA [57]. Free radical attack is established to be a critical initiator of several chronic diseases, including type 2 diabetes [58]. Whole grains contain a mass of antioxidant compounds, which have been shown to possess potent antioxidant activity [59]. Many soluble antioxidant compounds, including phenolic acids, tocopherols and flavonoids, are present in whole grains [60]. The highest phenolic acid content is present in corn ( $265 \mathrm{mg}$ gallic acid equivalents $/ 100 \mathrm{~g}$ ), followed by wheat (135 mg), oats (111 mg) and rice (95 mg) [61]. Ferulic acid, one of the most well studied phenolic acids, is shown to be a strong antioxidant by donating hydrogen atoms to free radicals [61, 62]. Carotenoids in whole grains include lutein, alpha-carotene, beta-carotene and beta-cyproxanthin, are found in the bran and germ layers of whole grains, can also serve as antioxidants [63]. Further, antioxidant capacity is inherent in insoluble grain fibers $[64,65]$. Phytic acid is common phytochemical in many whole grains, and is well known as an antioxidant that represses ironcatalyzed redox reactions $[66,67]$.

Processing affects the biological activities of food and their ingredients, and most of grains and their food products go through very exhaustive food processing route [66, 68-70] Vitamin E, is another common ingredient of whole grains is a potent antioxidant that protects cell membranes and inhibits the formation of nitrosamines [72,73], but it is almost wholly removed during the refining process of whole grains [71]. In addition, the toasting process that many whole grains undergo (e.g. toasting whole wheat bread) increases the antioxidant activity compared to the raw materials, and actually develops a similar activity as that of many fruits and vegetables [74]. However, it is important to note that although many potent antioxidant compounds have been identified in whole grains, and their individual effects have been reported, but studies elucidating which compounds provide the most potent and specific effects in the context of whole grains is not done very comprehensively.

\section{Anti-Obesity Effects}

Epidemiologically, whole grains have been shown to beneficially alter critical measures of obesity, such as weight, Body Mass Index (BMI) and waist circumference [75]. Jonnalagadda et al. reviewed the prospective studies that utilize diverse patient databases to conclude that consumption of whole grains is inversely correlated with weight, BMI and waist circumference [14]. Further, visceral adipose tissue volume is negatively correlated with intake of whole grains (3 ounceequivalent servings per day) in both men and women [76]. Overall, reduction in weight gain has been the central benchmark for many of the clinical studies conducted on whole grains and obesity [14], with the trend heading towards examining the role of whole grains in decreasing visceral adiposity. The recent evidences also suggest that in addition to the marked decreases in body weight are associated with whole grain intake and suggested that, whole grains may contribute to the redistribution of fats within the body [76]. Intervention studies, however, report the inconsistent results of beneficial effects of whole gains in reducing overweight and obesity [14]. Therefore, it is more critical to place the emphasis on placing refined grains versus whole grains as a central tenant in many of these studies, as therein lies the challenge for society as a whole.

Overall, however, the vast majority of clinical studies both epidemiological and mechanistic - are conducted in the Caucasian population. Studies exploring similar benefits in other populations both within the United States and around the world are warranted in more comprehensive manner and considering parallel comparison to existing studies designs.

The mechanisms by which phytochemicals present in whole grains directly affect various conditions of obesity have been minimally explored. One mechanism suggests that whole grains act to increase satiety, and consequently render those who consume whole grains to desire less food intake [77, 78]. Indeed, several studies have reported an increased feeling of fullness with the addition of whole grain to the diet $[77,78]$. These reductions in hunger caused by intake of whole grains may be due to a differential profile of gut hormones, as whole grains have been shown to influence ghrelin, peptide YY, glucagon-like peptide 1, cholecystokinin levels [14]. Although variability in changes in hunger versus satiety feelings in several studies have been reported, and acknowledged the subjects do not demonstrate a decrease in energy intake [14]. However, in some studies, whole grains have been clearly observed to reduce energy intake [79]. This calls for further research elucidating the mechanisms by which whole grains may alter satiety and food intake signals, a critical player in the delicate balance between energy intake and expenditure.

\section{Beneficial Effects on Cholesterol Metabolism}

Imbalance in cholesterol metabolism and elevated cholesterol levels are common conditions in obese and diabetic subjects, and tightly correlated with increased incidence of cardiovascular diseases [80, 81]. Phytosterols from whole grains have been shown to displace cholesterol from micelles, in turn reducing cholesterol absorption, increasing excretion, and ultimately decrease serum cholesterol in hyper cholesterolemic men and women [82-84]. Linoleic acid and palmitate, both part of the unsaturated fatty acids that are highly present in whole grain fats, and whole grain derived these fatty acids have been 
Table 2: Whole grain phytochemicals associated with diabetes and obesity

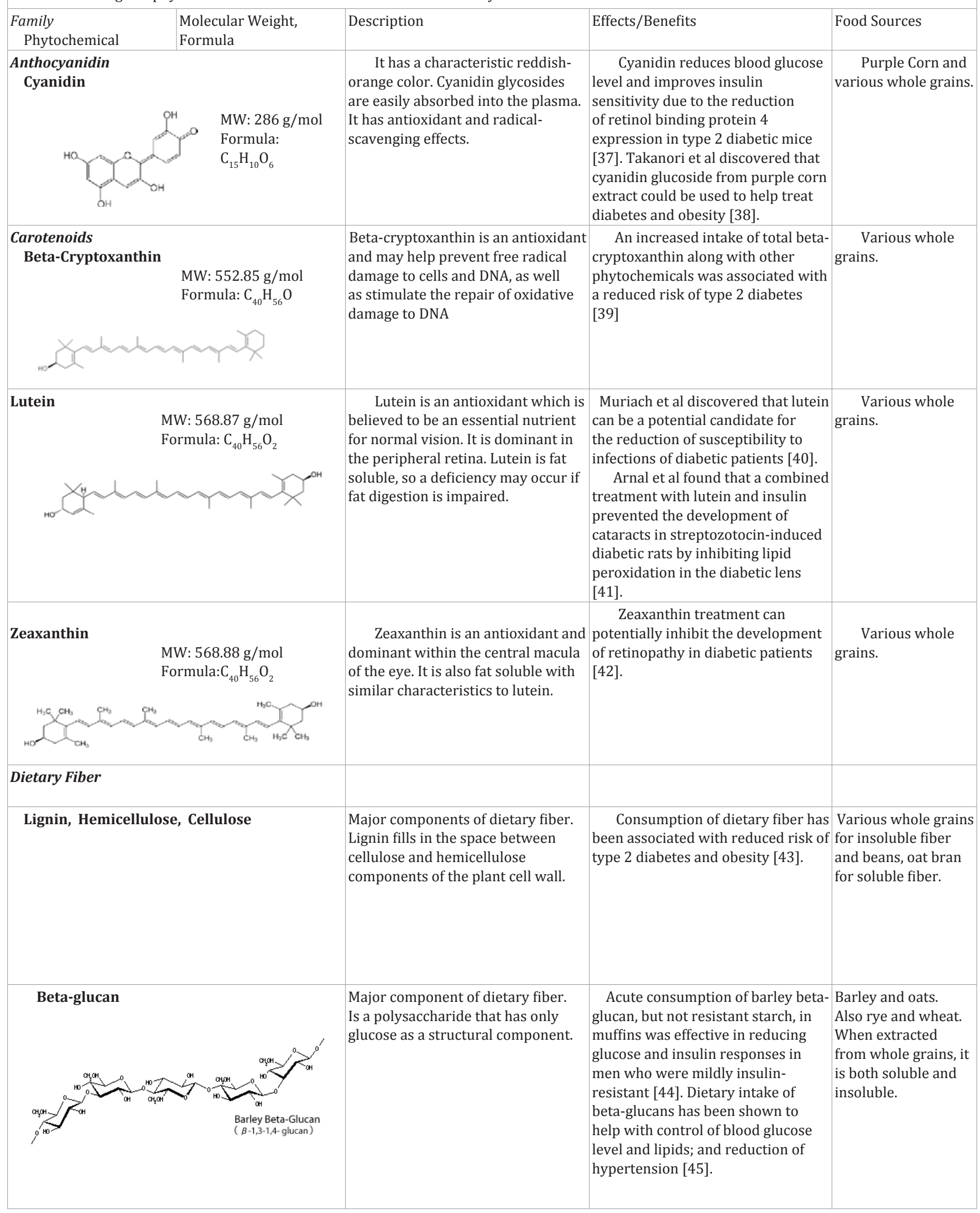




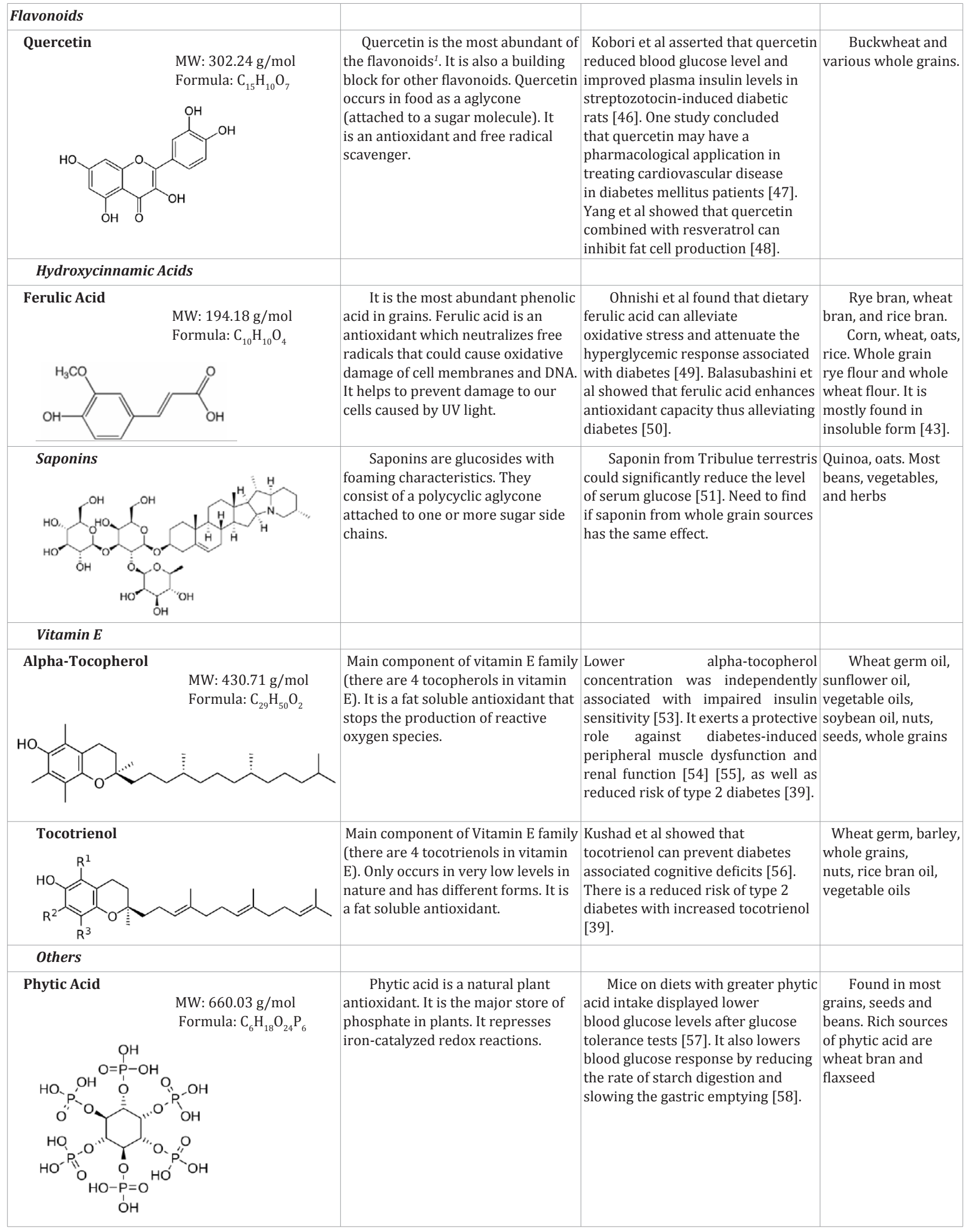




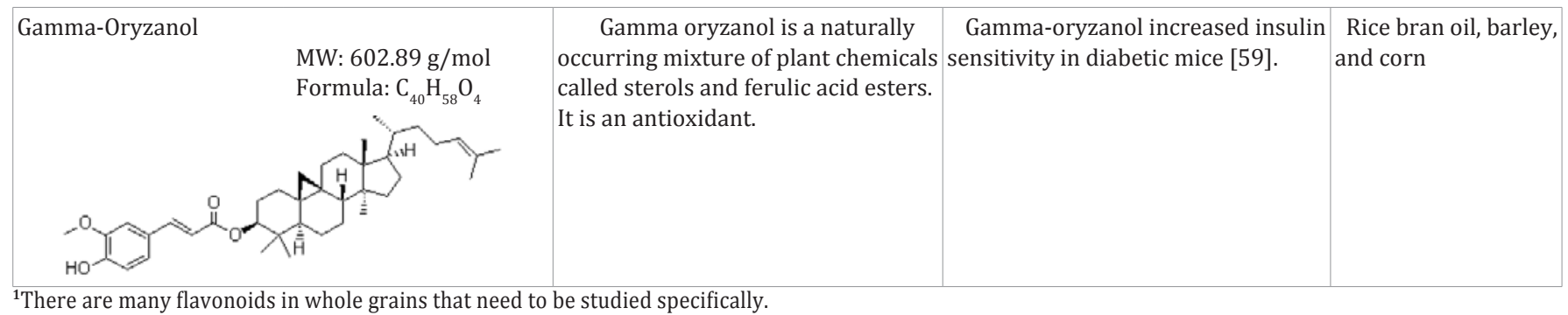

${ }^{1}$ There are many flavonoids in whole grains that need to be studied specifically.

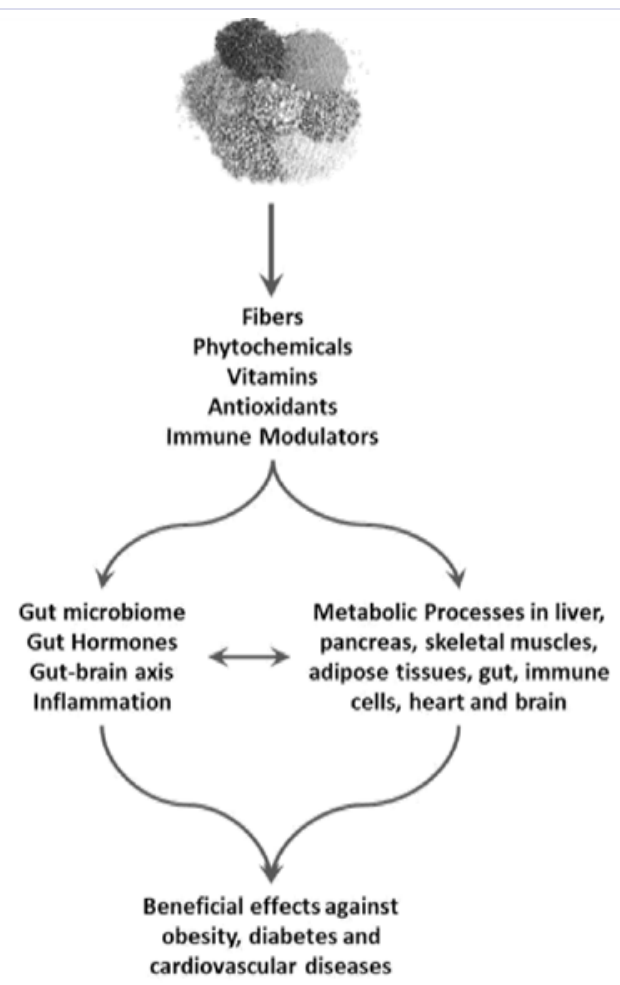

Figure 2: Beneficial potential of whole grains and their ingredients against metabolic diseases.

demonstrated to reduce serum cholesterol and increase Low Density Lipoprotein (LDL) [85-87]. Anti-nutrients discussed by Slavin et al, strongly suggested that beneficial effects of whole grain derived anti-nutrients modulated blood sugar levels along with lowering plasma cholesterol and triglycerides [25]. Maki et al showed that whole grain Ready To Eat (RTE) oat cereal consumption as part of a dietary program for weight loss had favorable beneficial impacts on fasting lipid levels and waist circumference in selected population [88]. Durrazo et al, described in a very well designed randomized clinical trial investigating the effect of intake of whole grain foods rich in lignans as part of an habitual diet showed a modest hypocholesterolemic effects [89]. Still, several studies have inconclusive results to show beneficial effects of whole grain foods on cholesterol metabolism, but the limitation in these studies are that many studies used processed whole grain foods and its known that food processing might have great impact on biological activities of whole grain as is. Further studies are needed to conclude, effects of whole grain foods, their ingredients and types of processing have impact of health beneficial effects.

\section{Whole Grain-Microbiome Axis}

Now It's very obvious that microbiome have major impact on human health and contribute in pathology of several human diseases including cancer, metabolic and immune disorders [90]. Initial original studies from Jeffery Gordon and others concluded that gut microbiome have major impact on obesity and type 2 diabetes development and contribute in regulation of whole body metabolic function of host [91]. Although, recent study couldn't find the direct correlation of gut microbiome with obesity from pooled data of more than 10 studies [92], but several independent studies have concluded not only correlation, rather direct impact of microbiome on several metabolic features i.e. body weight gain, fat mass, blood glucose and lipid levels [93]. Diet has major impact on gut microbiome and its derived metabolites to influence host metabolism by several mechanisms [94]. Dietary fibers are major source of gut microbiome derived metabolites i.e. short chain fatty acids that can influence several metabolic pathways i.e. gut hormone release, AMP activated kinases, and mitochondrial functions [95-97]. Effect of gut microbiome is not limited only into the gut but signal from microbiome sense by several other metabolic organs i.e. liver, adipose tissue, skeletal muscles, pancreas, and brain to regulate major energy homeostasis mechanisms [96-98]. Modulation of gut microbiome has been proposed one of the prominent strategies to counter metabolic diseases. Probiotics and prebiotics are major natural modulators of gut microbiome and have been known for several health beneficial effects to the host, including improvement in metabolic functions $[99,100]$. Whole grains have also been suggested to possess prebiotic effects, as fecal samples of humans fed with whole grain cereals contain significantly increased numbers of fecal bifidobacteria and lactobacilli [101]. Prebiotics and probiotics are increasingly being explored by many research groups worldwide, and are being established to play critical roles in the pathogenesis of obesity [99]. Connolly et al showed the impact of oat grain on the microbial ecology of the human gut and its potential to beneficially modulate the gut microbiota through increasing Bifidobacterium population by changing fermentation activity to produce short chain fatty acids i.e. propionate and butyrate [102]. Whole grains showed significant decreasing obesity parameters that correlated with changes in gut microbiome [103]. Whole grain feeding impacted energy intake that was associated with changes in metabolomics profile of gut microbiome derived metabolites and gut hormone changes [104]. Zhou et al clearly described that feeding of whole grains dominantly changes gut microbiome and improve insulin 
sensitivity and other metabolic measures in mice models [105]. Whole-grain barley feeding known to decrease the high fat dietinduced inflammation that is possibly related to formation of short chain fatty acids i.e. propionate, butyrate and changes in microbiota composition [106]. In addition, in this study authors found that high $\beta$-glucan content in the diet reduced plasma cholesterol levels [106]. Food processing is always a factor in whole grain induced changes in gut microbiome, as recent study showed that processing of whole-grain barley to barley malt have significant impact on gut microbiota and metabolites in rats fed high-fat diets [107]. As the mechanisms by which whole grains improve characteristics of overweight and obesity are for the most part obscure, it is critical for research to specifically explore the roles of phytochemicals and other constituents.

\section{Conclusions}

Whole grains contain a host of bioactive phyto-constituents i.e. fibers, alkaloids, flavonoids, saponins and other phytochemicals, vitamins, antioxidants and immune modulators, with known health benefits, including amelioration of chronic diseases (such as obesity and diabetes). These bioactive phytochemicals, both in isolation and in combination, appear to be key players in the improvement of glucose metabolism in different metabolic organs i.e. liver, pancreas, skeletal muscles, adipose tissue, gastrointestinal tract, immune cells and brain directly or indirectly via influencing gut microbiome, gut hormones, gutbrain axis and inflammatory response as well as oxidative stress and cholesterol profiles (Figure 2). However, the phytochemicals reviewed here warrant further in depth research. In current literature rarely any phytochemicals from whole grains are reported and discussed directly to influence metabolic function with Comprehensive and defined mechanism(s) of action. In summary, whole grains possess evident anti-diabetic and antiobese properties, but research must seek to further elucidate the role of both specific phytochemicals and establish mechanistic explanations for these effects. Further, there are unique subsets of phytochemicals in whole grains that are poorly characterized and purified. Therefore, chemical research is also essential to discover all of the bioactive components in whole grains, as each may have potent abilities to improve the pathophysiology of diabetes, obesity, and the resultant metabolic complications.

\section{References}

1. Hossain P, B Kawar, M El Nahas. Obesity and diabetes in the developing world--a growing challenge. N Engl J Med. 2007;356(3):213-215. DOI: 10.1056/NEJMp068177

2. Brown L, H Poudyal, SK Panchal. Functional foods as potential therapeutic options for metabolic syndrome. Obes Rev. 2015;16(11): 914-941. doi: 10.1111/obr.12313.

3. Khan MI, Anjum FM,Sohaib M,SAmeen A. Tackling metabolic syndrome by functional foods. Rev Endocr Metab Disord. 2013;14(3):287-297. doi: 10.1007/s11154-013-9270-8.

4. USDA, Dietary Guidelines for Americans 2005. , in USDA Human Nutrition Information Service Hyattsville , MD2005.

5. USDA, Dietary Guidelines for Americans; 20102010

6. Bachman JL, Reedy J ,Subar AF, Krebs-Smith SM. Sources of food group intakes among the US population, 2001-2002.J Am Diet Assoc. 2008;108(5):804-814. doi: 10.1016/j.jada.2008.02.026.

7. Kris-Etherton PM, Lefevre M,Mensink RP, Petersen B,Fleming J,Flickinger BD. Trans fatty acid intakes and food sources in the U.S. population: NHANES 1999-2002. Lipids. 2012;47(10):931-940. DOI:10.1007/s11745-012-3704-z.

8. Cleveland LE, Moshfegh AJ, Albertson AM,Goldman JD, Dietary intake of whole grains. J Am Coll Nutr. 2000;19(3 Suppl): 331S-338S.

9. Harnack L, SA Walters, DR Jacobs. Dietary intake and food sources of whole grains among US children and adolescents: data from the 19941996 Continuing Survey of Food Intakes by Individuals. J Am Diet Assoc. 2003;103(8):1015-1019. DOI:10.1053/jada.2003.50190.

10. de Punder K, L Pruimboom. The dietary intake of wheat and other cereal grains and their role in inflammation. Nutrients. 2013;5(3):771787. doi:10.3390/nu5030771.

11.AACC, AACC Members Agree on Definition of Whole Grain, in AACC1999: St. Paul, MN.

12. USFDA, FDA provides guidance on "Whole Grain" for manufacturers 2006.

13. Rebello CJ, FL Greenway, JW Finley. Whole grains and pulses: a comparison of the nutritional and health benefits. J Agric Food Chem. 2014;62(29):7029-7049. doi: 10.1021/jf500932z.

14. Jonnalagadda SS, Harnack L, Liu RH, Mc Keown N, Seal C, Liu S, et al. Putting the whole grain puzzle together: health benefits associated with whole grains--summary of American Society for Nutrition 2010 Satellite Symposium. J Nutr. 2011;141(5):1011S-7022S. doi: 10.3945/jn.110.132944

15. Adom KK, ME Sorrells, RH Liu. Phytochemicals and antioxidant activity of milled fractions of different wheat varieties. J Agric Food Chem. 2005;53(6):2297-2306. DOI:10.1021/jf048456d

16. USDA, USDA National Nutrient Database for Standard Reference. 2009. Nutrient Data Laboratory.

17. Liu S, Manson JE, Stampfer MJ, Hu FB, Giovannucci E, Colditz GA, et al. A prospective study of whole-grain intake and risk of type 2 diabetes mellitus in US women. Am J Public Health. 2000;90(9):1409-1415.

18. Liu S, Manson JE, Stampfer MJ, Rexrode KM, Hu FB, Rimm EB, et al. Whole grain consumption and risk of ischemic stroke in women: A prospective study. JAMA. 2000;284(12):1534-1540.

19. Schatzkin A, ParkY, Leitzmann MF, HollenbeckAR, Cross AJ. Prospective study of dietary fiber, whole grain foods, and small intestinal cancer. Gastroenterology. 2008;135(4):1163-1167. doi:10.1053/j. gastro.2008.07.015

20. Kastorini CM, DB Panagiotakos. Dietary patterns and prevention of type 2 diabetes: from research to clinical practice; a systematic review. Curr Diabetes Rev. 2009;5(4):221-227.

21. Priebe MG, van Binsbergen JJ, de Vos R, Vonk RJ. Whole grain foods for the prevention of type 2 diabetes mellitus. Cochrane Database Syst Rev. 2008;23(1):CD006061. doi:10.1002/14651858.CD006061.pub2.

22. Aune D, Norat T, Romundstad P, Vatten LJ. Whole grain and refined grain consumption and the risk of type 2 diabetes: a systematic review and dose-response meta-analysis of cohort studies. Eur J Epidemiol. 2013;28(11):845-858. doi:10.1007/s10654-013-9852-5.

23. Jenkins DJ, Wolever TM, Jenkins AL, Giordano C, Giudici S, et al. Low glycemic response to traditionally processed wheat and rye products: bulgur and pumpernickel bread. American Journal of Clinical Nutrition. 1986;43(4):516-520.

24. Heaton KW, Marcus SN, Emmett PM, Bolton CH. Particle size of wheat, 
maize, and oat test meals: effects on plasma glucose and insulin responses and on the rate of starch digestion in vitro. American Journal of Clinical Nutrition. 1988;47(4):675-682.

25. Slavin J. Whole grains and human health. Nutr Res Rev. 2004;17(1):99110. doi:10.1079/NRR200374.

26. Pereira MA, Jacobs DR Jr, Pins JJ, Raatz SK, Gross MD, Slavin JL, et al. Effect of whole grains on insulin sensitivity in overweight hyperinsulinemic adults. American Journal of Clinical Nutrition. 2002;75(5):848-855.

27. Sasaki R, Nishimura N, Hoshino H, Isa Y, Kadowaki M, Ichi T, et al. Cyanidin 3-glucoside ameliorates hyperglycemia and insulin sensitivity due to down regulation of retinol binding protein 4 expression in diabetic mice. Biochemical Pharmacology. 2007;74(11):1619-1627. DOI:10.1016/j.bcp.2007.08.008.

28. Tsuda T, Horio F, Uchida K, Aoki H, Osawa T. Dietary cyanidin 3-O-betaD-glucoside-rich purple corn color prevents obesity and ameliorates hyperglycemia in mice. J Nutr. 2003;133(7):2125-2130.

29.Zujko ME, Witkowska AM, Górska M, Wilk J, Krętowski A. Reduced intake of dietary antioxidants can impair antioxidant status in type 2 diabetes patients. Pol Arch Med Wewn. 2014;124(11):599-607.

30. Montonen J, Knekt P, Järvinen R, Reunanen A. Dietary antioxidant intake and risk of type 2 diabetes. Diabetes Care. 2004;27(2):362-366.

31. Arnal E, Miranda M, Almansa I, Muriach M, Barcia JM, Romero FJ, et al. Lutein prevents cataract development and progression in diabetic rats. Graefes Arch Clin Exp Ophthalmol. 2009;247(1):115-120.

32. Muriach M, Bosch-Morell F, Arnal E, Alexander G, Blomhoff R, Romero FJ. Lutein prevents the effect of high glucose levels on immune system cells in vivo and in vitro. J Physiol Biochem. 2008;64(2):149-157.

33. Burekovic A, A Dizdarevic-Bostandzic, A Godinjak. Poorly regulated blood glucose in diabetic patients-predictor of acute infections. Med Arh. 2014;68(3):163-166.

34. Davis TM, Weerarathne T, Foong Y, Mason C, Davis WA, et al Community-acquired infections in type 2 diabetic patients and their non-diabetic partners. The Fremantle Diabetes Study. J Diabetes Complications. 2005;19(5):259-263.

35. Kowluru RA, B Menon, DL Gierhart. Beneficial effect of zeaxanthin on retinal metabolic abnormalities in diabetic rats. Invest Ophthalmol Vis Sci. 2008;49(4):1645-1651.

36. Alminger M, C Eklund-Jonsson. Whole-grain cereal products based on a high-fibre barley or oat genotype lower post-prandial glucose and insulin responses in healthy humans. European Journal of Nutrition. 2008;47(6):294-300. doi:10.1007/s00394-008-0724-9.

37. Granfeldt Y, Liljeberg H, Drews A, Newman R, Björck I. Glucose and insulin responses to barley products: influence of food structure and amylose-amylopectin ratio. American Journal of Clinical Nutrition. 1994;59(5):1075-1082.

38. Behall K, Scholfield D, Hallfrisch J. Barley beta-glucan reduces plasma glucose and insulin responses compared with resistant starch in men. Nutrition Research. 2006;26(12):644-650.

39. Chen J, K Raymond K. Beta-glucans in the treatment of diabetes and associated cardiovascular risks. Vasc Health Risk Manag. 2008;4(6):1265-1272.

40. Kobori M, Masumoto S, Akimoto Y, Takahashi Y. Dietary quercetin alleviates diabetic symptoms and reduces streptozotocin-induced disturbance of hepatic gene expression in mice. Molecular Nutrition \& Food Research. 2009;53(7):859-868.

41. Li C, Zhang WJ, Frei B. Quercetin inhibits LPS-induced adhesion molecule expression and oxidant production in human aortic endothelial cells by p38-mediated Nrf2 activation and antioxidant enzyme induction. Redox Biol. 2016;9:104-113.

42. Chao CL, Hou YC, Chao PD, Weng CS, Ho FM. The antioxidant effects of quercetin metabolites on the prevention of high glucose-induced apoptosis of human umbilical vein endothelial cells. Br J Nutr. 2009;101(8):1165-1170. DOI:10.1017/S0007114508073637.

43. Yang JY, Della-Fera MA, Rayalam S, Ambati S, Hartzell DL, Park HJ, etal. Enhanced inhibition of adipogenesis and induction of apoptosis in 3T3L1 adipocytes with combinations of resveratrol and quercetin. Life Sciences. 2008;82(19-20):1032-1039. DOI:10.1016/j.lfs.2008.03.003.

44. Ohnishi M, Matuo T, Tsuno T, Hosoda A, Nomura E, Taniguchi H, et al. Antioxidant activity and hypoglycemic effect of ferulic acid in STZinduced diabetic mice and KK-Ay mice. Biofactors. 2004;21(1-4):315319.

45. Balasubashini MS, Rukkumani R, Viswanathan P, Menon VP. Ferulic acid alleviates lipid peroxidation in diabetic rats. Phytother Res. 2004;18(4):310-314.

46. Li M, Qu W, Wang Y, Wan H, Tian C. Hypoglycemic effect of saponin from Tribulus terrestris. Zhong Yao Cai. 2002; 25(6):420-422.

47. Illison VK, Rondó PH, de Oliveira AM, D’Abronzo FH, Campos KF. The relationship between plasma alpha-tocopherol concentration and vitamin $\mathrm{E}$ intake in patients with type 2 diabetes mellitus. Int J Vitam Nutr Res. 2011;81(1):12-20. DOI:10.1024/0300-9831/a000046.

48. Arnlöv J, Zethelius B, Risérus U, Basu S, Berne C, Vessby B. Serum and dietary beta-carotene and alpha-tocopherol and incidence of type 2 diabetes mellitus in a community-based study of Swedish men: report from the Uppsala Longitudinal Study of Adult Men (ULSAM) study. Diabetologia. 2009;52(1):97-105. DOI: 10.1007/s00125-008-1189-3.

49. Winterbone MS, Sampson MJ, Saha S, Hughes JC, Hughes DA. Prooxidant effect of alpha-tocopherol in patients with type 2 diabetes after an oral glucose tolerance test--a randomised controlled trial. Cardiovasc Diabetol. 2007;22(6):8. DOI:10.1186/1475-2840-6-8.

50. Hasan MY, Alshuaib WB, Singh S, Fahim MA. Effects of alphatocopherol on diabetes-induced alterations of synaptic transmission and contractile features in murine dorsiflexor muscle. Endocr Res. 2003;29(4):419-428.

51. Nascimento Gomes G, Barbosa FT, Radaeli RF, Cavanal MF, Mello Aires M, Zaladek Gil F. Effect of D-alpha-tocopherol on tubular nephron acidification by rats with induced diabetes mellitus. Braz J Med Biol Res. 2005;38(7):1043-1051. DOI:/S0100-879X2005000700007.

52. Kuhad A, Bishnoi M, Tiwari V, Chopra K. Suppression of NF-kappabeta signaling pathway by tocotrienol can prevent diabetes associated cognitive deficits. Pharmacol Biochem Behav. 2009;92(2):251-259. DOI:10.1016/j.pbb.2008.12.012.

53. LL Dilworth, FO Omoruyi, Simon OR, EY St A Morrison, HN Asemota. The effect of phytic acid on the levels of blood glucose and some enzymes of carbohydrate and lipid metabolism. West Indian Med. J. 2005;54(2):102-106. DOI.org/10.1590/S0043-31442005000200003.

54. Sung-Hyen Lee, Hong-Ju Park, Hye-Kyung Chun, So-Young Cho, SooMuk Cho, Hyun Soon Lillehoj. Dietary phytic acid lowers the blood glucose level in diabetic KK mice. Nutrition Research. 2006;26(9):474479 .

55. Yoon JH, Thompson LU, Jenkins DJ. Jenkins, The effect of phytic acid on in vitro rate of starch digestibility and blood glucose response. American Journal of Clinical Nutrition. 1983;38(6):835-842.

56. Cheng HH, Ma CY, Chou TW, Chen YY, Lai MH. Gamma-oryzanol ameliorates insulin resistance and hyperlipidemia in rats with 
streptozotocin/nicotinamide-induced type 2 diabetes. Int J Vitam Nutr Res. 2010;80(1):45-53. doi: 10.1024/0300-9831/a000005.

57. Giustarini D, Dalle-Donne I, Tsikas D, Rossi R. Oxidative stress and human diseases: Origin, link, measurement, mechanisms, and biomarkers. Crit Rev Clin Lab Sci. 2009;46(5-6):241-281. doi: 10.3109/10408360903142326.

58. Nikooyeh B, Neyestani TR. Neyestani, Oxidative stress, type 2 diabetes and vitamin D: past, present and future. Diabetes Metab Res Rev. 2016;32(3):260-267. doi: 10.1002/dmrr.2718.

59. Thompson LU. Antioxidants and hormone-mediated health benefits of whole grains. Crit Rev Food Sci Nutr. 1994;34(5-6):473-497.

60. Holst B, Williamson G. Williamson. Nutrients and phytochemicals: from bioavailability to bioefficacy beyond antioxidants. Curr Opin Biotechnol. 2008;19(2):73-82. doi: 10.1016/j.copbio.2008.03.003.

61. Adom KK, Liu RH. Antioxidant activity of grains. J Agric Food Chem. 2002;50(21):6182-6187.

62.Zieliński H, Kozłowska H. Kozlowska. Antioxidant activity and total phenolics in selected cereal grains and their different morphological fractions. J Agric Food Chem. 2000;48(6):2008-2016.

63. Hu QP, Xu JG. Profiles of carotenoids, anthocyanins, phenolics, and antioxidant activity of selected color waxy corn grains during maturation. J Agric Food Chem, 2011;59(5):2026-2033.

64. Celik EE, Gökmen V, Skibsted LH. Synergism between soluble and dietary fiber bound antioxidants. J Agric Food Chem. 2015;63(8):2338 2343

65. Saura-Calixto, F. Dietary fiber as a carrier of dietary antioxidants: an essential physiological function. J Agric Food Chem. 2011;59(1):4349. doi: 10.1021/jf1036596.

66. Rasane P, Jha A, Kumar A, Sharma N. Reduction in phytic acid content and enhancement of antioxidant properties of nutricereals by processing for developing a fermented baby food. J Food Sci Technol. 2015;52(6):3219-3234. doi: 10.1007/s13197-014-1375-x.

67. Graf E, Eaton JW. Antioxidant functions of phytic acid. Free Radic Biol Med. 1990;8(1):61-69.

68. Rodríguez-Roque MJ, de Ancos B, Sánchez-Vega R, Sánchez-Moreno C, Cano MP, Elez-Martínez P. et al. Food matrix and processing influence on carotenoid bioaccessibility and lipophilic antioxidant activity of fruit juice-based beverages. Food Funct. 2016;7(1):380-389. doi: 10.1039/c5fo01060h.

69. Bernaert N, De Loose M, Van Bockstaele E, Van Droogenbroeck B. Antioxidant changes during domestic food processing of the white shaft and green leaves of leek (Allium ampeloprasum var. porrum). Sci Food Agric. 2014;94(6):1168-1174. doi: 10.1002/jsfa.6389.

70. Kunyanga CN, Imungi JK, Okoth MW, Biesalski HK, Vadivel V. Antioxidant and type 2 diabetes related functional properties of phytic acid extract from Kenyan local food ingredients: effects of traditional processing methods. Ecol Food Nutr. 2011;50(5):452-471. doi: 10.1080/03670244.2011.604588.

71. Atkinson J, Epand RF, Epand RM. Tocopherols and tocotrienols in membranes: a critical review. Free Radic Biol Med, 2008;44(5): 739764. DOI:10.1016/j.freeradbiomed.2007.11.010.

72. Goufo P, Trindade H. Rice antioxidants: phenolic acids, flavonoids, anthocyanins, proanthocyanidins, tocopherols, tocotrienols, gamma-oryzanol, and phytic acid. Food Sci Nutr. 2014;2(2): 75-104 doi:10.1002/fsn3.86.
73. Sies H. Carotenoids and tocopherols as antioxidants and singlet oxygen quenchers. J Nutr Sci Vitaminol (Tokyo). 1992;27-33.

74. Dekker M, Verkerk R, van der Sluis AA, Khokhar S, Jongen WM. Analysing the antioxidant activity of food products: processing and matrix effects. Toxicol in Vitro. 1999;13(4-5):797-799.

75. Susan S Cho, Lu Qi, George C Fahey, David M Klurfeld. Consumption of cereal fiber, mixtures of whole grains and bran, and whole grains and risk reduction in type 2 diabetes, obesity, and cardiovascular disease. American Journal of Clinical Nutrition. 2013;98(2):594-619.

76. McKeown NM, Troy LM, Jacques PF, Hoffmann U, O’Donnell CJ, Fox CS. Whole- and refined-grain intakes are differentially associated with abdominal visceral and subcutaneous adiposity in healthy adults: the Framingham Heart Study. American Journal of Clinical Nutrition. 2010;92(5):1165-1171. doi: 10.3945/ajcn.2009.29106.

77. Schroeder N, Gallaher DD, Arndt EA, Marquart L. Influence of whole grain barley, whole grain wheat, and refined rice-based foods on short-term satiety and energy intake. Appetite. 2009;53(3):363369. doi: 10.1016/j.appet.2009.07.019.

78. Berti C Riso P, Brusamolino A, Porrini M. Effect on appetite control of minor cereal and pseudocereal products. Br J Nutr. 2005;94(5):850858.

79. Juntunen KS, Niskanen LK, Liukkonen KH, Poutanen KS, Holst JJ, Mykkänen HM. Postprandial glucose, insulin, and incretin responses to grain products in healthy subjects. American Journal of Clinical Nutrition. 2002;75(2):254-262.

80.Ding J, Reynolds LM, Zeller T, Müller C, Lohman K, Nicklas BJ et al. Alterations of a Cellular Cholesterol Metabolism Network Are a Molecular Feature of Obesity-Related Type 2 Diabetes and Cardiovascular Disease. Diabetes. 2015;64(10): 3464-3474. doi: $10.2337 / \mathrm{db} 14-1314$

81. FinlaysoN JS, Krook L, Larsson S. The effects of obesity, pyometra and diabetes mellitus on the fat and cholesterol contents of liver and spleen in the dog. Acta Physiol Scand. 1960;49:29-34. DOI:10.1111/j.1748-1716.1960.tb01925.x.

82. Hallikainen M, Kurl S, Laakso M, Miettinen TA, Gylling H. Plant stanol esters lower LDL cholesterol level in statin-treated subjects with type 1 diabetes by interfering the absorption and synthesis of cholesterol. Atherosclerosis. 2011;217(2): 473-478. doi: 10.1016/j. atherosclerosis.2011.03.041.

83. Plat J, Mensink RP. Plant stanol and sterol esters in the control of blood cholesterol levels: mechanism and safety aspects. Am J Cardiol. 2005;96(1A):15D-22D. 10.1016/j.amjcard.2005.03.015.

84. Fulayter J. Managing cholesterol with plant stanol esters. J Ren Nutr. 2002;12(3):E1-2. DOI:10.1053/jren.2002.33503.

85. Muka T, Bledar Kraja, Rikje Ruiter, Catherine E de Keyser, Albert Hofman, Bruno H Stricker. Dietary polyunsaturated fatty acids intake modifies the positive association between serum total cholesterol and colorectal cancer risk: the Rotterdam Study. J Epidemiol Community Health. 2016;70(9):881-887.

86. Abellan R, Mansego ML, Martínez-Hervás S, Morcillo S, Pineda-Alonso $\mathrm{M}$, Carmena $\mathrm{R}$, et al. Dietary polyunsaturated fatty acids may increase plasma LDL-cholesterol and plasma cholesterol concentrations in carriers of an ABCG1 gene single nucleotide polymorphism: study in two Spanish populations. Atherosclerosis. 2011;219(2):900-906. doi: 10.1016/j.atherosclerosis.2011.09.01

87. Wilke MS, MT Clandinin. Influence of dietary saturated fatty acids on the regulation of plasma cholesterol concentration. Lipids. 2005;40(12):1207-1213. 
88. Maki KC, Beiseigel JM, Jonnalagadda SS, Gugger CK, Reeves MS, Farmer $\mathrm{MV}$, et al. Whole-grain ready-to-eat oat cereal, as part of a dietary program for weight loss, reduces low-density lipoprotein cholesterol in adults with overweight and obesity more than a dietary program including low-fiber control foods. J Am Diet Assoc. 2010;110(2):205214. doi: 10.1016/j.jada.2009.10.037.

89. Durazzo A, Carcea M, Adlercreutz H, Azzini E, Polito A, Olivieri L, et al., Effects of consumption of whole grain foods rich in lignans in healthy postmenopausal women with moderate serum cholesterol: a pilot study. Int J Food Sci Nutr. 2014;65(5):637-645. doi: 10.3109/09637486.2014.893283

90. Althani AA, Marei HE, Hamdi WS, Nasrallah GK, El Zowalaty ME, Al Khodor S, et al. Human Microbiome and its Association With Health and Diseases. Journal of Cellular Physiology. 2016;231(8):1688-1694. doi: $10.1002 /$ jcp. 25284

91. Ley RE. Obesity and the human microbiome. Curr Opin Gastroenterol. 2010;26(1):5-11. doi: 10.1097/MOG.0b013e328333d751

92. Sze MA, PD Schloss. Looking for a Signal in the Noise: Revisiting Obesity and the Microbiome. MBio. 2016;7(4):e01018-16. doi: 10.1128/ mBio.01018-16

93. Franks I. Obesity: New light shed on obesity-associated gut microbiota. Nat Rev Gastroenterol Hepatol. 2011;8(10):537. doi: 10.1038/ nrgastro.2011.159.

94. Shoaie S, Ghaffari P, Kovatcheva-Datchary P, Mardinoglu A, Sen P, Pujos-Guillot E, et al., Quantifying Diet-Induced Metabolic Changes of the Human Gut Microbiome. Cell Metabolism. 2015;22(2):320331. doi: 10.1016/j.cmet.2015.07.001

95. Yan H, Hui Diao, Yi Xiao, Daiwen Chen, Bing Yu, Jun He, et al., Gut microbiota can transfer fiber characteristics and lipid metabolic profiles of skeletal muscle from pigs to germ-free mice. Sci Rep. 2016;6:31786.

96. Nehra V, Allen JM, Mailing LJ, Kashyap PC, Woods JA. Gut Microbiota: Modulation of Host Physiology in Obesity. Physiology (Bethesda). 2016;31(5):327-335. doi: 10.1152/physiol.00005.2016

97. Geach T. Gut microbiota: Antibiotics do not affect metabolism in obesity. Nat Rev Endocrinol. 2016;12(10):558. DOI: 10.1038/ nrendo. 2016.125

98. Bleau C, Karelis AD, St-Pierre DH, Lamontagne L. Crosstalk between intestinal microbiota, adipose tissue and skeletal muscle as an early event in systemic low-grade inflammation and the development of obesity and diabetes. Diabetes Metab Res Rev. 2015;31(6):545561. doi: 10.1002/dmrr.2617.
99. Barengolts E. Gut Microbiota, Prebiotics, Probiotics, and Synbiotics in Management of Obesity and Prediabetes: Review of Randomized Controlled Trials. Endocr Pract. 2016;22(10):1224-1234.

100. Yadav H, Lee JH, Lloyd J, Walter P, Rane SG. Beneficial metabolic effects of a probiotic via butyrate-induced GLP-1 hormone secretion. J Biol Chem. 2013;288(35):25088-25097. doi: 10.1074/ jbc.M113.452516.

101. Costabile A, Klinder A, Fava F, Napolitano A, Fogliano V, Leonard $C$, et al, Whole-grain wheat breakfast cereal has a prebiotic effect on the human gut microbiota: a double-blind, placebo-controlled, crossover study. Br J Nutr. 2008;99(1):110-120.

102. Connolly ML, JA Lovegrove, KM Tuohy. In vitro evaluation of the microbiota modulation abilities of different sized whole oat grain flakes. Anaerobe. 2010;16(5):483-488. doi: 10.1016/j. anaerobe.2010.07.001

103. Foerster J, Maskarinec G, Reichardt N, Tett A, Narbad A, Blaut M, et al. The influence of whole grain products and red meat on intestinal microbiota composition in normal weight adults: a randomized crossover intervention trial. PLoS One. 2014;9(10):e109606. doi: 10.1371/journal.pone.0109606.

104. Cooper DN, RJ Martin, NL Keim. Does Whole Grain Consumption Alter Gut Microbiota and Satiety? Healthcare (Basel). 2015;3(2):364392. DOI 10.3390/healthcare3020364

105. Zhou AL, Hergert N, Rompato G, Lefevre M.Whole grain oats improve insulin sensitivity and plasma cholesterol profile and modify gut microbiota composition in C57BL/6J mice. J Nutr. 2015;145(2):222-230. doi: 10.3945/jn.114.199778.

106. Zhong Y, Marungruang N, Fåk F, Nyman M. Effects of two wholegrain barley varieties on caecal SCFA, gut microbiota and plasma inflammatory markers in rats consuming low- and highfat diets. Br J Nutr. 2015;113(10):1558-1570. doi: 10.1017/ S0007114515000793

107. Zhong Y, M Nyman, F Fak. Modulation of gut microbiota in rats fed high-fat diets by processing whole-grain barley to barley malt. Molecular Nutrition \& Food Research. 2015;59(10):2066-2076. doi: 10.1002/mnfr.201500187

108. Hu BJ, Ya-Nan Hu, Song Lin, Wen-Jiang Ma, Xiao-Rong Li. Application of Lutein and Zeaxanthin in nonproliferative diabetic retinopathy. Int J Ophthalmol. 2011;4(3):303-306. doi: 10.3980/j.issn.22223959.2011.03.19

109. Okarter N, RH Liu. Health benefits of whole grain phytochemicals. Crit Rev Food Sci Nut. 2010;50(3):193-208. doi: 10.1080/10408390802248734. 